\title{
Design Sleep Mode and Wakeup Method for OpenFlow Switches
}

\author{
Tran Hoang V $\mathbf{u}^{1,2}, \mathrm{Vu}$ Cong Luc ${ }^{1}$ \\ ${ }^{1}$ Hanoi University of Science and Technology \\ ${ }^{2}$ Danang College of Technology, The University of Danang \\ Email : thvu@dct.udn.vn
}

Abstract- In this paper, we present a design and an evaluation of two power management modes that reduce the energy consumption of OpenFlow switches. First, we define two new low power modes: SLEEP PORT and SLEEP SWITCH, which reduce energy consumption in cases where packets on port or switches are absent. Second, we present a Wake on LAN (WOL) method for OpenFlow Switches to wake up Ethernet ports or the whole switch from inactive states. Finally, we describe our design, experimental results and performance evaluations. Our results show that the control SLEEP PORT mode on a switch might save about $9.8 \%$ power consumption per port, and up to about $60 \%$ of total power consumption of the switch with SLEEP SWITCH mode. In addition, we will implement this method to Openflow Switch bases on NetFPGA- 10 Gigabit in the future.

Keywords- OpenFlow Switch; NetFPGA; Low Power; Data Center Network; Green Networking.

\section{INTRODUCTION}

Power consumption in the ICT infrastructure is a pressing concern. Electricity used by data centers worldwide increased about $56 \%$ from 2005 to 2010 , accounting for between $1.1 \%$ and $1.5 \%$ of total electricity use [1]. Many system components in the data center contribute to the overall power consumption, including servers, power, cooling, storage, networking equipment, etc. Nowadays, many network devices have tended to integrate additional functionalities to sleep when not being used and automatically wake up when receiving requests. By shutting down wireless network card of a handheld device when no internet action is being taken place, the battery lifetime can be increased. In this case, the network card is powered only when an incoming call is received. This is known as "wake-on-wireless" [2]. Moreover, the IEEE 802.1X Wake on LAN (WOL) [3] supporting feature allows dormant PCs to be powered up when the switch receives a specific Ethernet frame, known as the "magic packet" [4].

Following this trend, some power management methods for networking devices have been proposed to reduce network power consumption, can be generally divided into two categories: the sleep mode supporting devices $[5,6]$ and rate adaptation mechanism supporting devices [7, 8]. Sleeping scheme powers off idle devices or components into sleep states [6] for a pre-estimated duration, and wakes up the sleeping devices or components when new packets arrive. However, this scheme is fragile against burst traffic [8]. In [9] and [10], a framework called ECODANE (Reducing Energy Consumption in Data Center Networks based on Traffic Engineering) ${ }^{1}$ was proposed. It focuses on optimizing the power consumption of network components by designing an intelligent network control system that dynamically adapts the set of active network components corresponding to the total traffic going through the data center. The experimental results in [9] and [10] have shown that by disabling unused links (i.e. ports) and switches, an energy saving of $25 \%$ to $40 \%$ can be achieved. In [11], a Clock Controller (CC) which receives control messages from an OpenFlow controller and adapts the working clock frequency of the switch according to traffic throughput to save energy was designed. Such a framework, however, requires the use of a more flexible and configurable network architecture such as Software-Defined Networking (SDN), in which the OpenFlow is one of

\footnotetext{
${ }^{1}$ Part of the work in this paper is done within the scope of the ECODANE research project which is co-sponsored by the Ministry of Science and Technology (Vietnam) and the Federal Ministry of Education and Research (Germany).
} 
the technologies widely used. There have been attempts to extend the current commercial switches to support OpenFlow protocols [12] [13] or to build a complete Openflow switch for research purposes [14]. However, these switches do not have power aware functionalities including SLEEP modes and WOL. In this paper, we propose methods for intelligently controlling the power consumption of OpenFlow switches used in data centers by adding SLEEP modes and WOL mechanism to the switches. The main contributions of our work are the following:

- We define two new sleep modes: SLEEP PORT mode and SLEEP SWITCH mode.

- We design a new WOL module for NetFPGA based OpenFlow Switches which can wake up Ethernet ports or switch when they are in a sleep mode.

The rest of the paper is organized as follows. Section II defines two new sleep modes for OpenFlow switches. Section III presents the design of the new WOL module for OpenFlow Switches. Section IV describes experimental results. Conclusions are drawn in section V.

\section{SLEEP MODES FOR OPENFLOW SWITCHES}

In [11] we designed a clock controller module integrated in the core of the NetFPGA based OpenFlow switch which can adjust the working clock frequency of the switch from $125 \mathrm{MHz}$ down to 3.90625 MHz as in Fig.1.

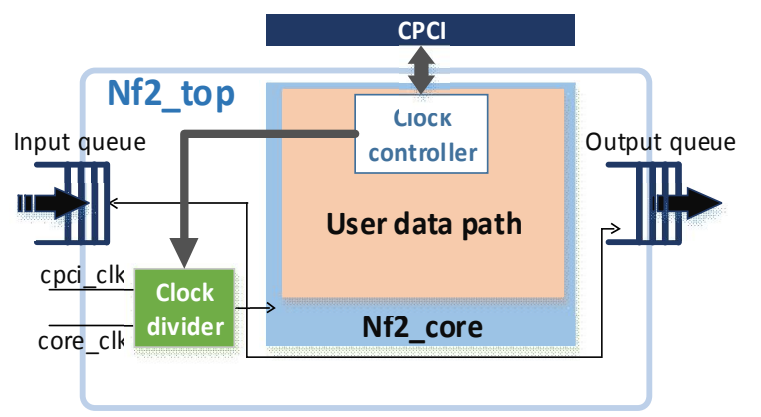

Figure 1. Structure of the frequency divider
We could also change the state of any port of the switch to idle mode by modifying some bits in the control register in the Enthernet control chip [19].

From these results we define two new sleep modes as follows.

- SLEEP PORT mode: This mode is used to turn off one or more Ethernet port when there is no traffic flowing through the ports. In this mode, the switch is running at $125 \mathrm{MHz}$ in order to maintain the operation of other Ethernet ports.

- SLEEP SWITCH mode: This mode is activated only when there is not any traffic going through any Ethernet port. In this case, the switch does not need to process the data flow and can be running at the minimum clock frequency of $3.90625 \mathrm{MHz}$, while three out of the four Ethernet ports are completely turned off. In this mode, switch still maintains the operation of one Ethernet port at $10 \mathrm{Mbps}$ so that the switch can be waken-up immediately to normal operation by WOL method.

\section{DESIGN NEW WOL MODULE FOR OPENLOW SWITCHES}

In Section II, we present two sleep modes for OpenFlow switches to save power consumption. In order to wake up a port or the whole switch from a sleep mode, we can send an OpenFlow control message to the switch. However, in many cases, sending OpenFlow control messages to wake up a number of switches in the network at the same time can take a long time. To resolve this problem, we propose to add a new WOL module to OpenFlow Switches with this new function, an Ethernet port or a switch can auto-wake up when they receive a broadcast packet (WOL packet) from other devices in the network.

\section{A. Wake On Lan packet}

WOL is hardware and software technology to wake-up sleeping systems by sending one or more special coded network packets to target machines which are equipped and enabled to respond to those packets. This additional functionality allows administrators to perform maintenance on systems 


\section{Research, Development on Information and Communications Technology}

even if the user has powered them down. The Wake on LAN feature allows the administrator to remotely power up all sleeping machines so they can receive updates [15]. The sending specific coded network packet is (are) called "WOL packet" or "Magic packet" $[5,15]$. Each device has its own unique WOL packet that consists of a header of the six bytes of all bits 1(0XFFFFFFFFFFFF) followed by the physical address of the device (48-bit MAC address, i.e. 0x112233445566) repeated 16 times for a total of 102 bytes as can be seen in Fig. 2 .

Base on this standard, two extended bytes are placed right after the 16 times repeats of the MAC address of the port that listens to the WOL packet. Those extended bytes for an OpenFlow switch are used to wake up Ethernet ports or switch from a sleep mode.

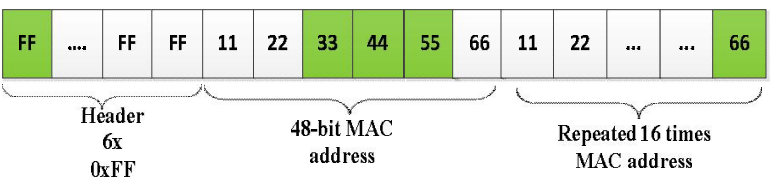

Figure 2. The standard WOL packet

The port can operate at four different states includes off and three states of link rate $10 \mathrm{Mpbs} /$ $100 \mathrm{Mpbs} / 1 \mathrm{Gbps}$. Therefore, each 2-bit group is used to control working modes of an Ethernet port. Besides, two extra bits are needed to change the operating frequency of the switch. Fig. 3 shows the bit descriptions of two extended bytes.

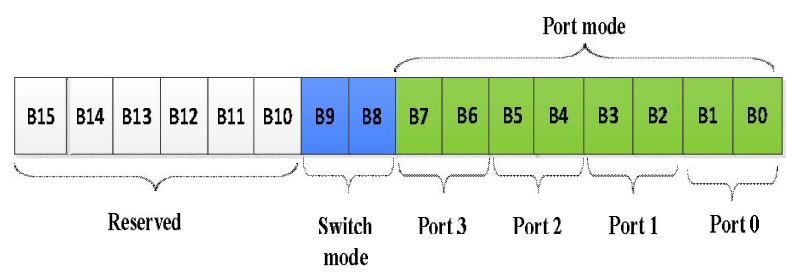

Figure 3. Bit meanings of two extended bytes of WOL packet for OpenFlow Switches

Table 1. states of port 0

\begin{tabular}{|c|c|}
\hline $\mathbf{b}_{\mathbf{1}} \mathbf{b}_{\mathbf{0}}$ & State of port \\
\hline 00 & off \\
\hline 01 & $10 \mathrm{Mbps}$ \\
\hline 10 & $100 \mathrm{Mbps}$ \\
\hline 11 & $1 \mathrm{Gbps}$ \\
\hline
\end{tabular}

To change the working modes of an Ethernet port, packet sender will set two bits corresponding to the target port. Table 1 shows all possible value of bit group $\left\{b_{1}, b_{0}\right\}$ that can be used to change the state of port 0 . Other ports can be easily be controlled in a similar manner by setting bits group: $\left\{b_{3}, b_{2}\right\}$ for port $1,\left\{b_{5}, b_{4}\right\}$ for port $2,\left\{b_{7}, b_{6}\right\}$ for port 3 .

Moreover, two bits $\left\{b_{9}, b_{8}\right\}$ contain values to change the operating frequency of the switch, as shown in Table 2.

Table 2. Change operating frequency of switch

\begin{tabular}{|c|c|}
\hline $\mathbf{b}_{\mathbf{9}} \mathbf{b}_{\mathbf{8}}$ & Operating Frequency \\
\hline 00 & $3.90625 \mathrm{MHz}$ \\
\hline 01 & $125 \mathrm{MHz}$ \\
\hline
\end{tabular}

Any port of the available ports of an OpenFlow switch can be used as listening port that can wait for the magic packet and wake itself or other ports up . The controller only needs to know which port is the listening port and can control the whole switch via this port. This method also provides a flexible way to manage a network of devices. Before being put into sleep mode, a switch can send a message back to the controller to tell it which port will be kept running at $10 \mathrm{Mbps}$. The controller receives all feedback from devices and create a backup route that can be used to wake up a switch either directly or via running switches.

\section{B. Design Wake On Lan function for Openflow} Switch

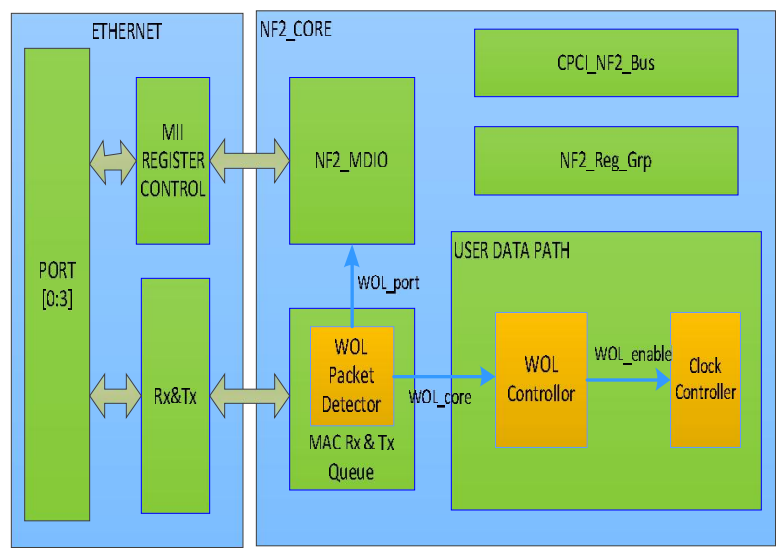

Figure 4. WOL Packet Detector block. 
In $[15,16]$, it is known that to enable WOL function, the network adapter must stay power on even if the PC is shut down. The BIOS must be configured to accept the WOL signal and the operating system/network driver must be configured to keep the network adapter powered on and the motherboard responsive to the WOL signal. Similarly, when a switch operates at the SLEEP SWITCH mode, one Ethernet port still runs at 10 Mbps to listen to a WOL packet. At the MAC RX \& TX queue blocks, we build a WOL packet detector block that detects the WOL packet. As shown in Fig.4, the output signals of this block are connected to the NF2 MDIO block that controls the SLEEP PORT mode and to the WOL Controller that controls the Sleep Switch mode.

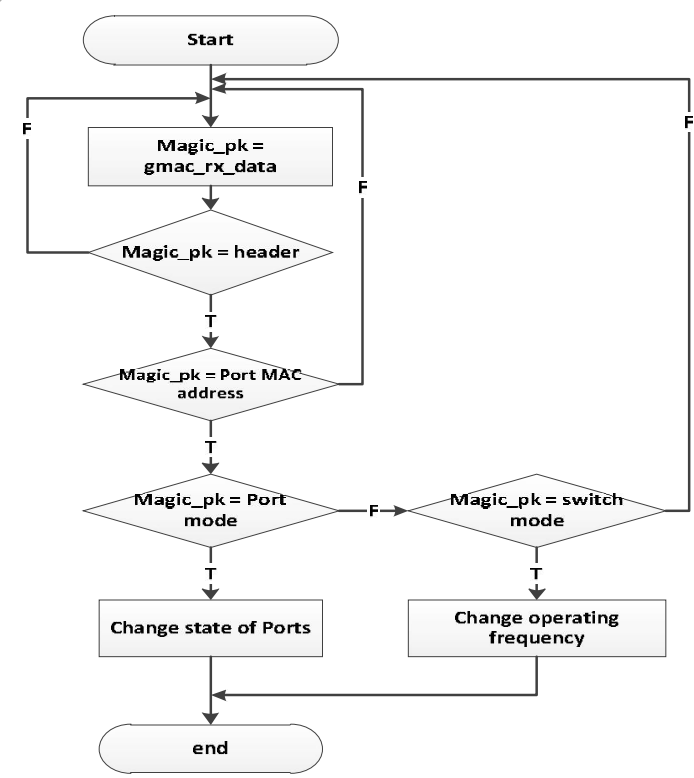

Figure 5. WOL Packet Detector block working principle

When a WOL packet is sent to the switch by other devices on LAN network through the listening Ethernet port, it will be recognized over the bytes header. This packet is compared with the MAC address of the listening port. If it is the same, WOL packet is continuously compared with port mode and switch mode located at the extended bytes defined in part A. WOL Packet Detector block will send WOL_port signal that corresponds to the port. This signal is connected to the NF2_MDIO block that can change the status of the port. We toggle the $11^{\text {th }}$ bit of the MII register [11] to ' 1 ' to turn-on the port. And we use a combination of the $6^{\text {th }}$ bit and the $13^{\text {th }}$ bit to change the bandwidth on each port.

Besides, WOL Packet Detector block also sends WOL core signal to the WOL Controller block to control the Clock Controller block which is presented in paper [11]. This block will change the operating frequency of the switch to $3.90625 \mathrm{MHz}$ or to $125 \mathrm{MHz}$. The working principle of the WOL Packet Detector is shown in Fig.5.

\section{EXEPERIMENTAL RESULTS}

\section{Power of Switch at two sleep modes}

In order to measure saving power consumption at the two sleep modes defined in Section II, we build a hardware test-bed including a NOX Controller, and an OpenFlow Switch based on NetFPGA-1G board as in Fig.6.

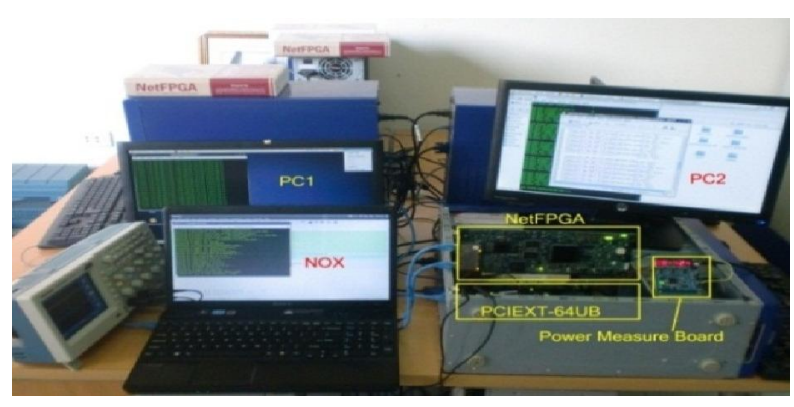

Figure 6. Test-bed system for measurement.

The NOX controller version 1.0.0 is implemented on a host PC running Ubuntu version 10.10. OpenFlow switch version 1.0.0.4 based on NetFPGA version 3.0.1 developed by Stanford [17] is used. We also use $\mathrm{PC} 1$ and $\mathrm{PC} 2$ to generate packets on links. $\mathrm{PC} 1$ and $\mathrm{PC} 2$ will exchange data at hard load of bandwidth. Oscilloscope and Power Measure Board are used to read $\mathrm{ADC}$ value at the test-point $3.3 \mathrm{~V}$ and 5.0V via PCIEXT-64UB [18] and then display, and calculate the power consumed.

Measurement results of the power consumption of a NetFPGA based OpenFlow switch with two different sleep modes (i.e.: SLEEP PORT mode and SLEEP SWITCH mode) are shown in Table 3. 


\section{Research, Development on Information and Communications Technology}

In paper [11], we measured the total energy consumption of the switch when all four ports operate at a bandwidth of around $1 \mathrm{Gbps}$ and we obtained the result of $11580^{\circ} \mathrm{mW}$. This is the largest power consumption of the switch under normal operation. With the proposed sleep modes, the switch can save about $9.8 \%$ of the total power consumption when one port is in the SLEEP PORT mode and up to about $60 \%$ if the switch is put to the SLEEP SWITCH mode,

Table 3. OpenFlow switch Power consumption at different sleep modes

\begin{tabular}{|l|c|}
\hline \multicolumn{1}{|c|}{ Mode } & Power Consumption of Switch (mW) \\
\hline Sleep one port & 10434 \\
\hline Sleep two ports & 9299 \\
\hline Sleep three ports & 8203 \\
\hline Sleep switch & 4576 \\
\hline
\end{tabular}

\section{Test Wake On Lan method for Openflow switch}

We also design the other test-bed system to wake up switch when it runs at sleep modes by other (another) PC.(Fig.7)

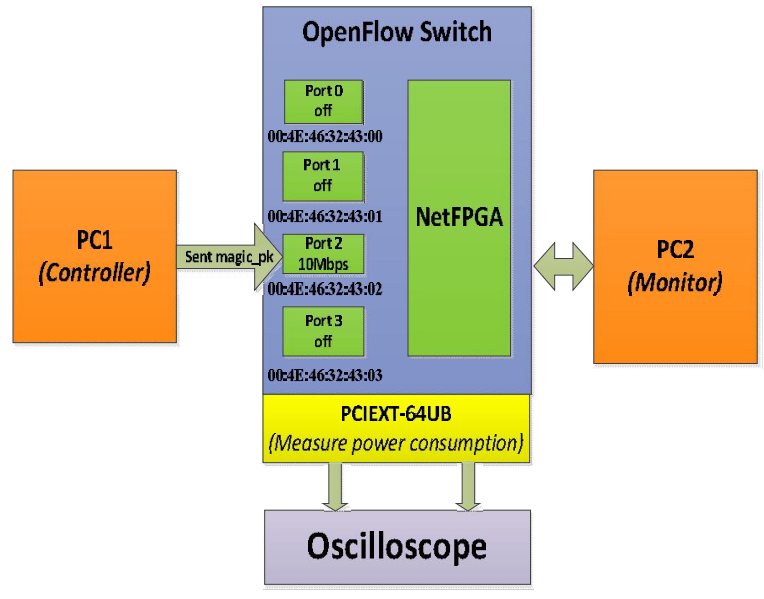

Figure 7. Testbed system for Wake On Lan function

As shown in Fig.7, PC1 plays the role of a controller which has a packet generator to send the WOL packet to the Ethernet port in order to wake up target OpenFlow switch operating in sleep modes. PC2 is used to supervise the working modes of the OpenFlow switch under test.
In our experiment, first, port 0 is turned off, and then a WOL packet is sent to port 2 which is working normally and listening to the WOL packet to wake up port 0 . This packet contains the MAC address of the Ethernet port 2 and has the $\left\{b_{0}, b_{1}\right\}$ bits set to " 11 ". As depicted in Fig. 8 after port 2 receives a WOL packet, port 0 is waken up successfully.

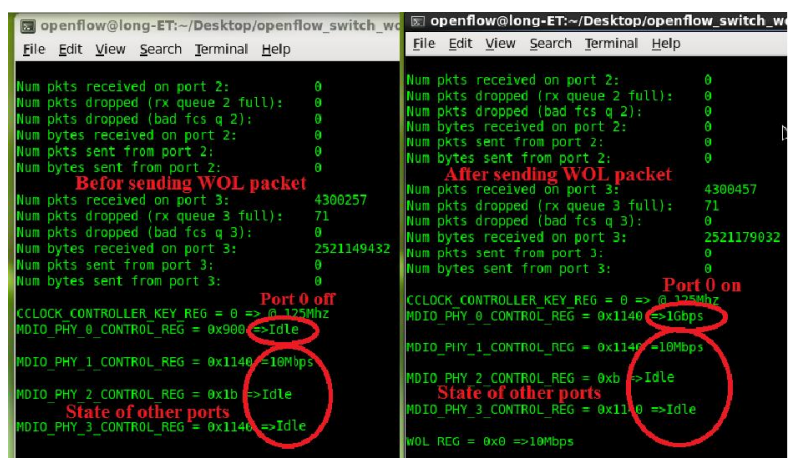

Figure 8. Monitor states of switch when wakes up port 0

In the second stage of the test, the OpenFlow switch is set to work in SLEEP SWITCH mode at which switch runs at frequency of $3.90625 \mathrm{MHz}$ and three ports are turned off, port 2 is running at 10 Mbps to listen the WOL packet. Then PC1 sends the WOL packet to wake the switch up to normal operation. This packet contains the MAC address of port 2 and has extended bytes as shown in Fig.9.

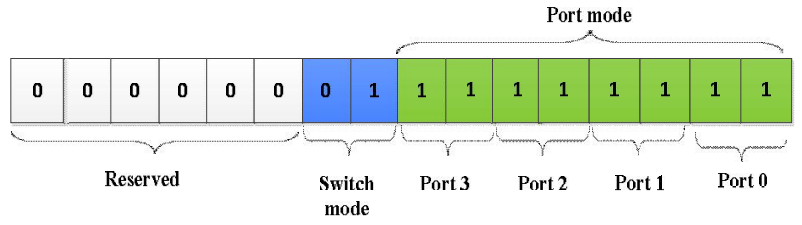

Figure 9. Extended bytes to wake up switch

After port 2 receives the WOL packet, the switch is waken up successfully and operate in normal state. PC2 also captures all monitored states of the switch, as shown in Fig. 10. 


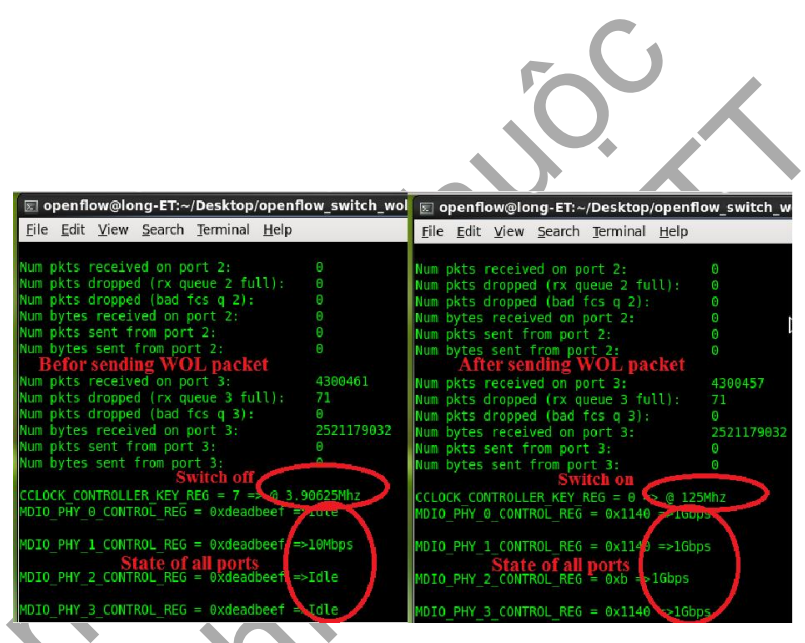

Figure 10. Monitor the waking up of the switch from the SLEEP SWITCH mode

\section{E. Proposed solution in the Future}

In the future, our group will implement this solution to NetFPGA-10G [20]. The 10G NetFPGA platform provides $4 \times 10$ Gigabit Ethernet SFP+ interfaces which are operated by AEL2005 PHYs, and two Samtec expansion connectors which are operated by 20 GTX dual transceivers. The PHYs are connected to a Xilinx Virtex-5 TX240T and communicate to XGMAC cores on the FPGA in full duplex mode.

Because this version of OpenFlow Switch has bandwidth $10 \mathrm{Gig} / 1$ port that consumes power higher than OpenFlow Switch $1 \mathrm{Gig}$, we strongly believe that our method will save a large amount of energy for OpenFlow Switch.

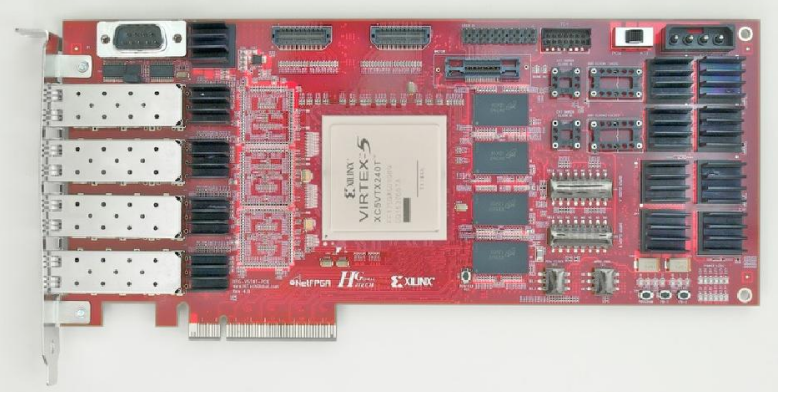

Figure 11. NetFPGA-10G

\section{CONCLUSTIONS AND FUTURE WORKS}

In this paper, we have defined and implemented two sleep modes for OpenFlow Switches to reduce power consumption. We have also implemented WOL method to wake up an NetFPGA based OpenFlow Switch from sleep modes to normal state. We also built the test-bed system and precisely measured the energy of the whole switch saving of about $9.8 \%$ power consumption per port, and up to about $60 \%$ of total power consumption of the switch with SLEEP SWITCH mode.

In the future, we will implement this method to Openflow Switch $10 \mathrm{Gig}$ and use in the design of power aware commercial OpenFlow Switches.

\section{REFERENCES}

[1]. Growth in data center electricity use 2005 to 2010 , http://www.analyticspress.com/datacenters.html

[2]. M. Gupta, "A feasibility study for power management in lan switches," in Proceedings of the 12th IEEE International Conference on Network Protocols, 2004.

[3]. Eugene Shih, Paramvir Bahl, Michael J. Sinclair, "Wake on wireless: An Event Driven Energy Saving Strategy for Battery Operated Devices."

[4]. "IEEE 802.1X Wake in LAN Support" Authentication Services Configuration Guide Cisco IOS Release 15MT.

[5]. "Magic Packet Technology" White paper,combinations thereof are trademarks of Advanced Micro Devices.

[6]. M.Gupta and S. Singh,"Dynamic ethernet link shutdown for energy conservation on ethernet links," in IEEE International Conference on Communications, 2007.

[7]. C. Gunaratne, K.Christensen, B.Nordman, and S. Suen, "Reducing the energy consumption of ethernet with adaptive link rate (alr)," IEEE Transactions on Computers, vol. 57, pp. 448-461, 2008.

[8]. S. Nedevschi, L. Popa, G. Iannaccone, S. Ratnasamy, and D. Wetherall, "Reducing network energy consumption via sleeping and rate-adaptation," in Proc. of NSDI, Berkeley, CA, USA: USENIX Association, pp. 323-336, 2008.

[9]. T.T. Huong, P.N. Nam, N. H. Thanh, D. Schlosser, M. Jarschel, R. Pries, "ECODANE - Reducing Energy Consumption in Data Center Networks based on Traffic Engineering" (poster), in the Proceedings of 11th Würzburg Workshop on IP: Joint ITG and EuroNF Workshop "Visions of Future Generation Networks" (EuroView2011), Würzburg, Germany, August 1st - 2nd 2011.

[10]. N.H. Thanh, P.N. Nam, T.T. Huong, N.T. Hung, L.K. Doanh and R. Pries, "Enabling Experiments for Energy-Efficient Data Center Networks on OpenFlow-based Platform", in the Proceedings of the 4th International Conference on Communications and Electronics 2012 (ICCE 2012), Hue, Vietnam, pp. 239-244, August 1st - 3rd 2012. 


\section{Research, Development on Information and Communications Technology}

[11]. T.H.Vu, T. Thanh, V. Q. Trong, N.H. Thanh, P.N. Nam "Energy Saving for OpenFlow Switch on the NetFPGA platform Using Multi-Frequency" in International Journal of Computing and Network Technology, No.1, pp.9-15, 1 Jan 2014.

[12]. "New HP 5400R zl2 Switch Series" http://h17007.www1.hp.com/us/en/networking/produ cts/switches/index.aspx\#.U-dORP1_tmU

[13]. "IBM System Networking RackSwitch G8264" http://www-03.ibm.com/systems/ networking/switches/ rack/g8264/index.html

[14]. John W. Lockwood, Nick McKeown, Greg Watson, Glen Gibb, Paul Hartke, Jad Naous, Ramanan Raghuraman, and Jianying Luo "NetFPGA- An Open Platform for Gigabit-rateNetwork Switching and Routing" IEEE International Conference on Microelectronic Systems Education, June 3-4, 2007 Sa Diego, CA.

[15]. White paper: Wake on Lan Technology. Leiberman Software Corporation. June 1, 2006.

http://www.liebsoft.com/pdfs/Wake_On_LAN.pdf

\section{AUTHORS' BIOGRAPHIES}

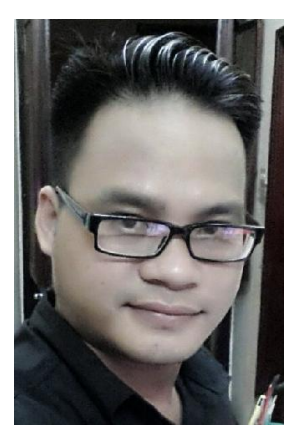

Tran Hoang Vu received B. Eng. degree in Electronics and Telecommunications from $\mathrm{Da}$ Nang University of Technology and M.Sc. degree from the University of Danang (Vietnam) in 2004 and 2007, respectively. From 2010 till 2015, he pursued his Ph.D. career at HUST. From 2004 until now he has been working at Danang College of Technology-The University of Danang, Vietnam. His research interests include Reducing power consumption of Data Center Networks, reconfigurable embedded systems and lowpower embedded system design.
[16]. Data Synergy TechNote: Wake On Lan Explained. 2011.

http://www.datasynergy.co.uk/products/wakeman/pdf s/Wake-on-LANExplained.pdf http://www.datasynergy.co.uk/products/wakeman/pdf s/Wake-onLANExplained.pdf/

[17]. Glen Gibb, John W. Lockwood, Jad Naous, Paul Hartke, and Nick McKeown "NetFPGA - An Open Platform for Teaching How to Build Gigabit-rate Network Switches and Routers" IEEE Transactions on Education, 2008.

[18]. "Product Specifications and User Manual of PCIEXT64U/UB and PCIeEXT-16HOT" v1r08 - April 12, 2013, Ultraview Corporation.

[19].http://wiki.netfpga.org/foswiki/NetFPGA/OneGig/Rel eases.

[20].http://netfpga.org/10G_specs.html

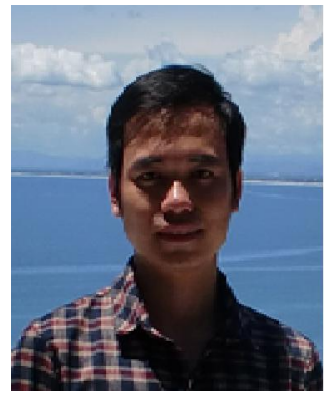

Vu Cong Luc is a student in School of Electronics and Telecommunications, Hanoi University of Science and Technology, Vietnam, where he has been since 2009. Since 2011 he has been working at the Embedded System and Reconfigurable Computing Lab, HUST.

His research interests include Reducing power consumption of Data Center Networks, reconfigurable embedded. 